$$
\text { Acta Cryst. (2008). A64, C202 }
$$

\section{Direct space and simultaneous direct-reciprocal space optimization models for phasing structures}

Alexander B. Smith ${ }^{1}$, Nikolaos V. Sahinidis ${ }^{2}$

${ }^{1}$ University of Illinois at Urbana-Champaign, Chemical and Biomolecular Engineering, 620 Whitney Ave, Pittsburgh, PA, 15221, USA, ${ }^{2}$ Carnegie Mellon University, 5000 Forbes Ave, Pittsburgh, PA, 15213, USA, E-mail:absmith2@uiuc.edu

The phase problem has recently been approached via combinatorial optimization techniques and the resulting Sieve method has been demonstrated to be effective for phasing centrosymmetric structures [1]. The purpose of the current work is to develop a more robust model for accurate phasing in the presence of odd triplets, and more important, to provide a combinatorial optimization approach to phasing non-centrosymmetric crystals. Two mixed-integer linear programming models for phasing are proposed; both of which include the introduction of specific direct space constraints with one additionally operating in reciprocal space. Direct space is constrained through sampling of electron density on a grid. Structure factors are calculated at these points in terms of the integer variables, which describe the phases. Bounds are then formulated using experimental data. Computational results are presented for a variety of structures. References

[1] A. B. Smith, H. Xu, and N. V. Sahinidis. An integer minimal principle and triplet sieve method for phasing centrosymmetric structures. Acta Crystallographica A, 63(2):164 - 171, 2007.

Keywords: phasing methods, algorithms, direct methods

\section{P02.02.07}

Acta Cryst. (2008). A64, C202

\section{Macromolecular structure solution by charge flipping}

Arie Van Der Lee $^{1}$, Christian Dumas ${ }^{2}$

${ }^{1}$ Institut Europeen des Membranes, cc047 Universite de Montpellier II, Montpellier, Herault, 34000, France, ${ }^{2}$ Centre de Biochimie Structurale, CNRS UMR5048, INSERM U554, F-34090 Montpellier, France, E-mail : avderlee@univ-montp2.fr

The solution of the crystallographic phase problem continues to be a challenge for the development of new and robust approaches. The recently discovered charge flipping phasing algorithm has received a growing interest in small-molecule crystallography and powder diffraction. This computational methodology radically differs from the classical direct methods as it neither requires a priori knowledge of space-group symmetry nor chemical composition and does not rely on probabilistic phase relations or a statistical framework. We show here that the charge flipping algorithm is capable to solve ab initio large macromolecular structures, up to $\sim 4,000$ atoms in the asymmetric unit, using suitable normalized intensity data at atomic resolution (1.1 $\AA$ or better). Moreover, we demonstrate that this algorithm also provides a very efficient tool for the determination of complex anomalously-scattering heavy-atom substructures at medium to low resolution (down to $5 \AA$ ). With the present extension to macromolecular crystallography charge flipping proves to be a very performing and general phase recovery algorithm in all fields of kinematical diffraction.

Keywords: charge flipping, macromolecular structure determination, sub-structure determination

\section{Direct observation of structural phase in CBED patterns - applications to structure determination}

Joanne Etheridge, Philip N Nakashima, Alexander F Moodie Monash University, Monash Centre for Electron Microscopy, Monash Centre for Electron Microscopy, Monash University, Victoria, 3800, Australia, E-mail:joanne.etheridge@mcem.monash.edu.au

It has been shown that the signs of three-phase invariants of a centrosymmetric structure can be observed directly by inspection of 3-beam convergent beam electron diffraction (CBED) patterns [1-5]. This ability to read-off phases directly and quickly from diffraction patterns suggests a new approach to the determination of structures that begins with knowledge of structure factor phases, rather than magnitudes. As demonstrated by Lonsdale [6], almost 80 years ago, knowledge of structure factor phases alone can be sufficient to determine outline structures. However, the difficulty in measuring phase has traditionally meant that structure determinations start from a knowledge of measured magnitudes, not phases. In this paper, we demonstrate practically how the signs of three-phase invariants can be observed directly from 3 beam CBED patterns using corundum as an example. (We will also describe how the corresponding structure factor magnitudes can be determined from the measurement of distances in the same 3 beam patterns.) We demonstrate how these observations of three-phase invariants alone can be applied using Lonsdale's simple, powerful and unambiguous approach to structure solution [6], to determine an outline of the structure of corundum.

[1] J. Gjonnes and R. Hoier A25 (1969) 595.

[2] A.F. Moodie, Chem. Scripta 14 (1978-79), 21

[3] A.F. Moodie, J. Etheridge and C.J. Humphreys, Acta Cryst. A52 (1996), 596.

[4] P.N.H. Nakashima, A.F. Moodie and J. Etheridge, Acta Cryst. A63 (2007), 387.

[5] P.N.H. Nakashima, A.F. Moodie and J. Etheridge, Ultramicroscopy (2008), in press.

[6] K. Lonsdale, Proc. R. Soc. 123A (1929), 494.

[7] The authors thank the Australian Research Council (ARC DP0346828).

Keywords: phase problem, structure solution, electron diffraction

\section{P02.04.09}

Acta Cryst. (2008). A64, C202-203

\section{Studies for S-SAD method using various wavelength at} SPring-8 and SAGA-LS

Masahide Kawamoto $^{1}$, Nobutaka Shimizu ${ }^{2,3}$, Seiki Baba ${ }^{2}$,

Kunio Hirata $^{3}$, Koutaro Ishiji ${ }^{1}$, Kazushi Sumitani ${ }^{1}$,

Toshihiro Okajima ${ }^{1}$, Hiroyuki Motoshima ${ }^{4}$, Keiichi Watanabe ${ }^{4}$,

Takashi Kumasaka ${ }^{2,3}$, Masaki Yamamoto ${ }^{3}$

${ }^{1}$ Kyushu Synchrotron Light Research Center, Beamline Group, 8-7 Yayoigaoka, Tosu-shi, Saga, 841-0005, Japan, ${ }^{2} J a p a n$ Synchrotron Radiation Research Institute, 1-1-1 Kouto, Sayo-cho, Sayo-gun, Hyogo 679-5198, Japan, ${ }^{3}$ RIKEN SPring-8 Center, Harima Institute, 1-1-1 Kouto, Sayo-cho, Sayo-gun, Hyogo, 679-5198, Japan, ${ }^{4}$ Department of Applied Biochemistry and Food Science, Faculty of Agriculture, Saga University, 1 Honjo-machi, Saga-city, Saga 840-8502, Japan, E-mail : kawamoto@saga1s.jp

Single-wavelength anomalous dispersion (SAD) method using sulfur atoms as an anomalous dispersion atom (S-SAD) is a powerful tool for the phase determination in protein crystallography. There are two 
strategies to collect dataset efficiently for the S-SAD method, (i) lowering the measurement error using high brilliant short wavelength $\mathrm{X}$-ray, (ii) augmenting the anomalous signal using long wavelength $\mathrm{X}$-ray. To validate these strategies, we experimented on the S-SAD method with standard samples at two synchrotron beamlines, SPring-8/BL41XU and SAGA-LS/BL15. (i)We have collected datasets from standard samples (insulin, thaumatin and lysozyme) using various wavelength between 0.71 to $1.90 \AA$ at the SPring- 8 undulator beamline BL41XU. We succeeded in the determination of the phases and building the molecular models of insulin from dataset collected in $0.9-1.9 \AA$ wavelength, thaumatin from $1.5-1.9 \AA$ and lysozyme from $1.5-1.7 \AA$, respectively. In addition, it ascertained that the dataset collected in longer wavelength allowed the phase determination and model building from lesser redundancy. (ii) SAGA-LS/BL15 has a bending magnet as a light source, and it is able to use X-ray between 0.54 to $5.90 \AA$ wavelengths. The preliminary X-ray diffraction experiment of insulin crystals using 1.5, 2.3, 2.6 and $2.9 \AA$ wavelengths was performed at this beamline. The $R_{\text {merge }}$ of the collected datasets were about $4-7 \%$ in all wavelengths, and the molecular models could be built automatically from dataset in 1.5 and $2.3 \AA$ wavelengths by S-SAD analysis. From the datasets collected using 2.6 and $2.9 \AA$ wavelengths, we could determine the position of sulfurs and the phase but not build their models automatically due to the less of the resolution.

Keywords: protein crystallography with synchrotron radiation, anomalous dispersion methods, sulfur

\section{P02.04.10}

Acta Cryst. (2008). A64, C203

\section{Sulphur SAD (S-SAD) phasing using $\mathrm{CoK}_{a}$ radiation}

Tomokazu Hasegawa, Akihito Yamano

PharmAxess, Inc., Biohills 308, 7-7-18, Saito-Asagi, Ibaraki, Osaka, 5670085, Japan, E-mail:tomokazu@pharmaxess.com

The S-SAD phasing is extremely useful for structure determination of a novel protein especially when its heavy atom derivatives are difficult to prepare. For S-SAD phasing, $\mathrm{Cr} K_{a}$ radiation has generally been used in a laboratory because its wavelength $(2.2909 \AA)$ is closer to the absorption edge of sulphur $(\sim 5 \AA)$ than that of $\mathrm{Cu} K_{a}$ radiation. However, precise measurements of anomalous signals using X-rays with such a long wavelength is sometimes difficult due to errors originating from numerous sources such as air dispersion, absorptions by solvent around a crystal, a mounting loop, and components of an instrument such as a window material. Furthermore, with a plate detector, it is cumbersome to collect high resolution data that are necessary to reach the final structure. One can minimize the camera length to record high angle reflections, however those reflections will suffer severe errors by absorption and obliqueness. The optimal wavelength for S-SAD phasing may lay somewhere in-between $\mathrm{Cu} K_{a}$ and $\mathrm{Cr} K_{a}$. Therefore we have developed an X-ray generator producing strong $\mathrm{Co}_{a}$ radiation (1.7902 $\AA$ ) based on an FR-E Suberbright (Rigaku) and built a S-SAD system around it. The final system consists of an FR-E Superbright DW $(\mathrm{Cu} / \mathrm{Co})(\mathrm{Rigaku})$, a VariMax Co (Rigaku Americas) and an R-RAXIS VII/HTC (Rigaku). To reduce the absorption, we eliminated the black shielding film of the image plate detector. In order to evaluate the Co system, we tried structure analysis of four commercially available proteins: Insulin, HEW-Lysozyme, Glucose isomerase, Trypsin and Thaumatin. We will show these experimental data at the conference.

Keywords: SAD, protein phasing, single anomalous diffraction
P02.05.11

Acta Cryst. (2008). A64, C203

\section{Development of computer software for general area detector diffraction system(GADDS)}

\section{Hohyuk Kim$^{1}$, Hosung Kim ${ }^{2}$}

${ }^{1}$ Chonnam National University, Dept. of Materials and Engineering, B-109 B27(College of Engneering Bldg) 300 Yongbong-dong, Buk-gu, Gwangju, Gwangju, 500-757, Korea (S), ${ }^{2}$ Chonnam National University, 418 B27(College of Engneering Bldg) 300 Yongbong-dong, Buk-gu, Gwangju, Gwangju, 500-757, Korea (S), E-mail:michita@nate.com

We have developed the software which determines the orientation of the single crystal using General Area Detector Diffraction System(GADDS). The developed software is possible to determines the orientation for all kinds of crystal system and to analyze the single crystal when Detector's position was laid down at every directions against the beam direction. In case a uncertainty of the position of diffraction spots is below a millimeter, the orientation can be determined the right way by the least squares fitting. The software can previously forecast a diffraction situation by inputting the data which are a crystal system, a lattice constant and suchlike. The software offers a method that turns the single crystal determined the orientation toward the direction wanted by user. GADDS Analysis Program developed with $\mathrm{C}++$ programming language can be utilized for the instruments of X-ray diffraction as well as Analysis of LaueFilm.

Keywords: Laue film, diffraction pattern, analysis software

\section{P02.05.12}

Acta Cryst. (2008). A64, C203-204

\section{Structural changes of reaction centre from Bl. viridis revealed by time-resolved Laue diffraction}

Annemarie B. Woehri ${ }^{1}$, Gergely Katona ${ }^{2}$, Emelie Fritz ${ }^{2}$, Erik Malmerberg ${ }^{2}$, Richard Neutze ${ }^{2}$

${ }^{1}$ Chalmers University of Technology, Department of chemical and biological Engineering, Medicinaregatan 9E, Lundberg Laboratory, Gothenburg, Vaestergoetland, 41390, Sweden, ${ }^{2}$ Department of Chemistry, Biochemistry and Biophysics, University of Gothenburg, SE-405 30 Gothenburg, Sweden, E-mail:wohri@chembio.chalmers.se

Reaction centre of Bl. viridis (RCvir) was the first membrane protein from which high resolution diffraction data was ever obtained. The structure showed three subunits and the photosynthetic pigments which were ordered in a two fold symmetric arrangement. Further, an electron pathway across the membrane was proposed, initiated by capturing a photon by the special pair, a bacteriochlorophyll dimer located on the periplasmic side. This absorption generates an electron, which migrates through the membrane to the movable ubiquinone, the final electron acceptor. After reduction to ubiquinol, it will then be released into the membrane. Even though the basic structure of RCvir is known, detailed structural information about how light gets converted into chemical energy is still missing. This lacking information is of major interest, since RCvir has high homology to photosytem II of higher plants. If one gets more insight into the photocycle of RCvir one could use this information and apply it directly to eukaryotic systems. One way of exploring the reaction path is to perform a time-resolved laue experiment where the photocycle is initiated with a laser and electron transport is monitored with a polychromatic x-ray beam. The illumination is very demanding for the crystal and thus robust crystals are an absolute requirement for the experiment. So, we crystallized RCvir using the sponge phase crystallization method and a type I crystal form 